CORRECTION

\title{
Correction to: The methyltransferase PRMT6 attenuates antiviral innate immunity by blocking TBK1-IRF3 signaling
}

Hua Zhang, Chaofeng Han, Tianliang Li, Nan Li and Xuetao Cao

Cellular \& Molecular Immunology (2020) 17:192; https://doi.org/10.1038/s41423-019-0337-7

Correction to: Cellular \& Molecular Immunology (2019) 16, 800-809; https://doi.org/10.1038/s41423-018-0057-4, published online 4 July 2018

In the published version of Fig. 2d, the HE image for Prmt6 ${ }^{+/+}$ mice of the medium group was mistakenly presented. Figure $2 d$ has now been corrected. The corrected version of Figure 2 is shown below.

Although we regret our mistake during figure assembly and would like to apologize for any inconvenience it may have caused, we did not manipulate our data in any way. This unintentional error also has no bearing on the work's scientific conclusions.
A

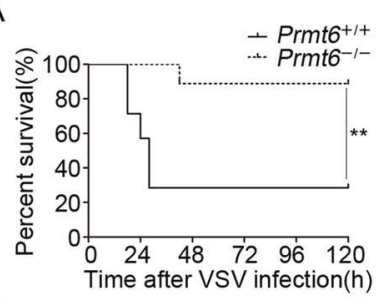

C
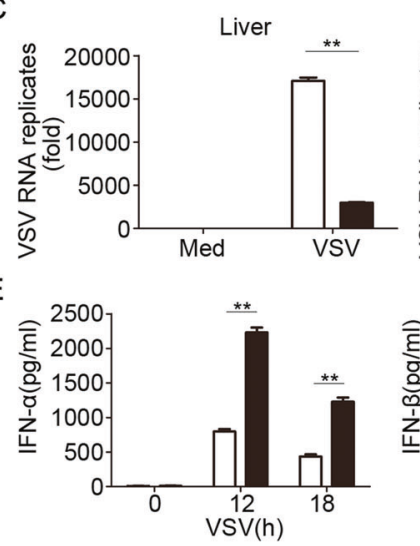
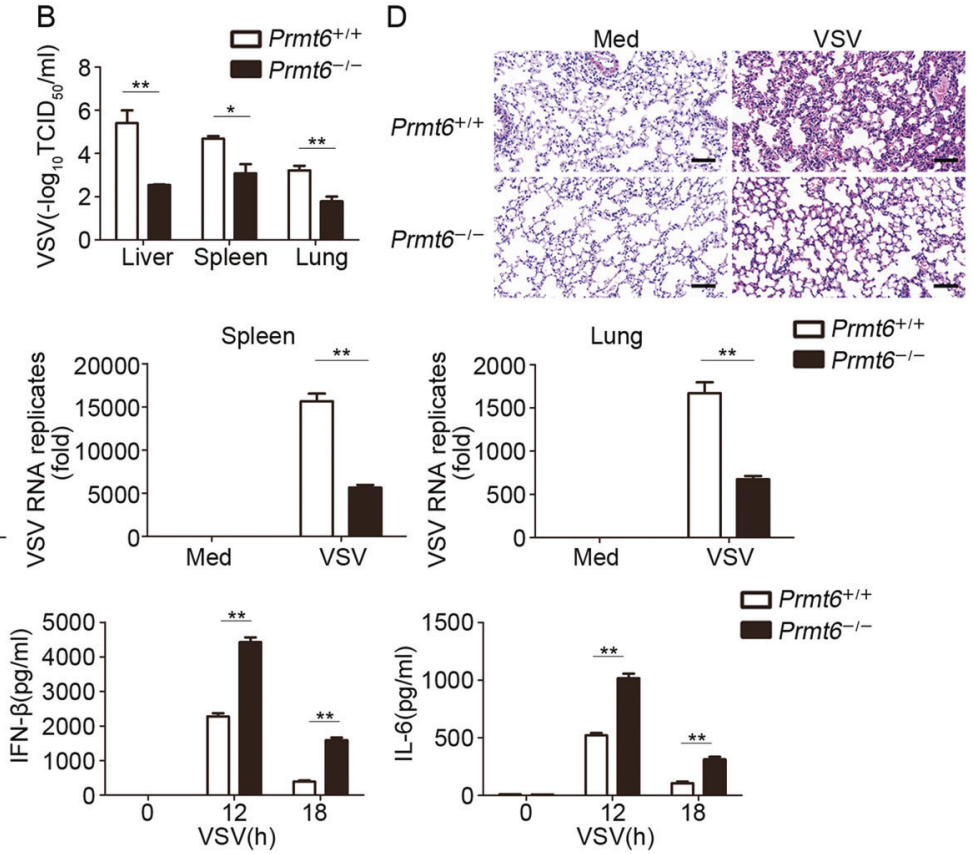

Fig. 2 